IJMMS 25:1 (2001) 43-52

PII. S0161171201003994

http://ijmms.hindawi.com

(C) Hindawi Publishing Corp.

\title{
THE INITIAL BOUNDARY VALUE PROBLEM OF A MIXED-TYPED HEMIVARIATIONAL INEQUALITY
}

\author{
GUO XINGMING
}

(Received 20 May 1999)

ABSTRACT. A mixed-typed differential inclusion with a weakly continuous nonlinear term and a nonmonotone discontinuous nonlinear multi-valued term is studied, and the existence and decay of solutions are established.

2000 Mathematics Subject Classification. Primary 34G20, 35M10, 35M20.

1. Introduction. In the present paper, the following initial boundary value problem of a degenerate multi-valued hyperbolic-parabolic inequality will be considered:

$$
\begin{gathered}
\ddot{u}(t)+A(t)(\dot{u})(t)+B(u)(t)+\varphi(u(x, t)) \ni f(t), \quad \text { a.e. } t \in[0, T], \\
u(x, t)=0, \quad \text { a.e. }(x, t) \in \Sigma=\partial \Omega \times[0, T], \\
u(0)=u_{0}, \quad \dot{u}(0)=u_{1},
\end{gathered}
$$

where $A$ is a weakly continuous operator; $B$ is a linear, continuous, and symmetric operator; $\varphi$ is a nonmonotonous, discontinuous, and nonlinear set-valued mapping.

Physical motivations for studying inequality (1.1) come partly from problems of continuum mechanics and optimal control problems, where nonmonotone, nonlinear, discontinuous, and multi-valued constitutive laws and boundary or external constraints lead to various typed hemivariational inequalities, the mixed hyperbolic-parabolic hemivariational inequality is one of those $[11,12,14]$.

For inequality (1.1), its stationary problems have been studied by many researchers (see $[1,2,4,13,14,15]$ and references therein). When $\varphi$ degenerates into a class of single-valued mappings, inequality (1.1) becomes an equation, and when $A$ and $B$ were some special linear mappings and satisfy some conditions, equation (2.1) and some of its evolution equations have been investigated and applied intensively (see $[5,3,6,7,8,9,10]$ and the references therein).

In this paper, we investigate the existence and decay of weak solution of the mixed hyperbolic-parabolic inequality (1.1) with $\varphi, A$, and $B$ satisfying some conditions. We apply the Faedo-Galerkin method for the proof of existence of solutions.

2. Preliminaries. Let $\Omega$ be a bounded open set of $\mathbb{R}^{n}$ with regular boundary $\Gamma$. Let $T$ denote a positive real number, $Q=\Omega \times[0, T]$. Suppose that $b \in L_{\mathrm{loc}}^{\infty}(\mathbb{R})$, for every $\rho>0$, set

$$
\underline{b}_{\rho}(\xi)=\operatorname{essinf}_{\left|\xi_{1}-\xi\right|<\rho} b\left(\xi_{1}\right), \quad \bar{b}_{\rho}(\xi)=\operatorname{esssup}_{\left|\xi_{1}-\xi\right|<\rho} b\left(\xi_{1}\right),
$$


they are all monotone for $\rho>0$. Set

$$
\underline{b}_{\rho}(\xi)=\lim _{\rho \rightarrow 0^{+}} \underline{b}_{\rho}(\xi), \quad \bar{b}(\xi)=\lim _{\rho \rightarrow 0^{+}} \bar{b}_{\rho}(\xi), \quad \varphi(\xi)=[\underline{b}(\xi), \bar{b}(\xi)] .
$$

Let $J(\xi)=\int_{0}^{\xi} b(t) d t$, then $\partial^{C} J(\xi) \subseteq \varphi(\xi)$, where $\partial^{C} J(\xi)$ denotes the Clarkesubdifferential of $J$ (see [2]). If $b\left(\xi_{ \pm}\right)$exists for every $\xi \in \mathbb{R}$, then $\varphi(\xi)=\partial^{C} J(\xi)$. If $b$ is continuous at the point $\xi$, then $\varphi(\xi)$ is single-valued at $\xi$, if $J$ is convex, $\varphi(\xi)$ is maximal monotone (see [2]).

Let $V=H_{0}^{1}(\Omega),(\cdot, \cdot)$ denotes the inner product of $L^{2}(\Omega),\langle\cdot, \cdot\rangle$ denotes the dual pair between $V$ and $V^{\prime}=H^{-1}(\Omega)$ which is compatible with the inner product of $L^{2}(\Omega)$. Let $|x|_{X}$ denote the norm of the element $x$ of the Banach space $X$.

Considering the following initial boundary value problem of a hyperbolic-parabolic hemivariational inequality:

$$
\begin{gathered}
\ddot{u}(t)+A(t) \dot{u}(t)+B u(t)+g(t)=f(t), \quad \text { a.e. } t \in[0, T], \\
u(x, t)=0, \quad \text { a.e. }(x, t) \in \Sigma=\partial \Omega \times[0, T], \\
u(0)=u_{0}, \quad \dot{u}(0)=u_{1}, \\
g(x, t) \in \varphi(u(x, t)), \quad \text { a.e. }(x, t) \in Q_{T}=\Omega \times[0, T],
\end{gathered}
$$

where $f, u_{0}$, and $u_{1}$ are given.

First we list some assumptions:

(1) $\exists c>0,|b(\xi)| \leq c(1+|\xi|)$, a.e. $\xi \in \mathbb{R}$.

(2) $A: L^{2}\left(0, T ; L^{2}(\Omega)\right) \rightarrow L^{2}\left(0, T ; L^{2}(\Omega)\right)$ is weakly continuous, and $A(t)$ is nonnegative, that is, $\langle A(t) v, v\rangle \geq 0$, for a.e. $t \geq 0$ and every $v \in L^{2}(\Omega)$.

(3) The function $t \rightarrow\langle A(t) u, v\rangle$ is measurable on $[0, T]$ for all $u, v \in L^{2}(\Omega)$.

(4) $B: H_{0}^{1}(\Omega) \rightarrow H^{-1}(\Omega)$ is linear, continuous, symmetric, and semicoercive, that is, $\exists c_{1}>0, c_{2}>0, c_{3}>0$

$$
\begin{gathered}
|B v|_{H^{-1}(\Omega)} \leq c_{1}|v|_{H_{0}^{1}(\Omega)}, \quad\langle B u, v\rangle=\langle B v, u\rangle, \quad \forall u, v \in H_{0}^{1}(\Omega), \\
\langle B v, v\rangle+c_{3}|v|_{L^{2}(\Omega)}^{2} \geq c_{2}|v|_{H_{0}^{1}(\Omega)}^{2}, \quad \forall v \in H_{0}^{1}(\Omega) .
\end{gathered}
$$

Let $\beta$ be any mollifier satisfying $\beta \in C^{\infty}(\mathbb{R}), \beta \geq 0$, supp $\beta \subset(-1,1)$, and $\int_{R} \beta(\xi)$ $d \xi=1$. Set

$$
b_{\varepsilon}(\xi)=\frac{1}{\varepsilon} \int_{\mathbb{R}} \beta\left(\frac{\xi-z}{\varepsilon}\right) b(z) d z=\int_{|z| \leq 1} \beta(z) b(\xi-\varepsilon z) d z, \quad \text { for every } \varepsilon>0 .
$$

It is easy to see that $b_{\varepsilon}$ is a smooth function, and also satisfies assumption (1) with possible different constant $c$ if $b$ is agreeable with assumption (1). For convenience, we denote $b_{1 / n}$ by $b_{n}$ for any positive integer $n$. 


\section{Existence of solution}

THEOREM 3.1. Assume that $f \in L^{2}\left(0, T ; L^{2}(\Omega)\right), u_{0} \in H_{0}^{1}(\Omega) \cap L^{p+1}(\Omega), u_{1} \in L^{2}(\Omega)$. Then, under assumptions (1), (2), (3), and (4), there exists a function $u$ defined in $\Omega \times$ $[0, T]$ such that

$$
\begin{gathered}
u \in L^{\infty}\left(0, T ; H_{0}^{1}(\Omega)\right) \bigcap C\left([0, T] ; L^{2}(\Omega)\right), \\
\dot{u} \in L^{\infty}\left(0, T ; L^{2}(\Omega)\right) \bigcap C\left([0, T] ; H^{-1}(\Omega)\right), \\
\ddot{u} \in L^{2}\left(0, T ; H^{-1}(\Omega)\right),
\end{gathered}
$$

and

$$
\begin{gathered}
\ddot{u}(t)+A(t) \dot{u}(t)+B u(t)+g(t)=f(t), \quad \text { in } L^{2}\left(0, T ; H^{-1}(\Omega)\right), \\
g(t) \in \varphi(u(x, t)), \quad \text { a.e. }(x, t) \in \Omega \times[0, T], \\
u(0)=u_{0}, \quad \dot{u}(0)=u_{1} .
\end{gathered}
$$

Proof. Let $\left\{e_{n}\right\}_{n=1}^{\infty}$ be a subset of $V=H_{0}^{1}(\Omega)$ satisfying $\overline{\operatorname{span}\left\{e_{n}\right\}}=V,\left(e_{i}, e_{j}\right)=$ $\delta_{i j}$. Let $x_{n}=\sum_{1}^{n} \omega_{i}^{1} e_{i} \rightarrow u_{0}$ strongly in $V$ and $L^{p+1}(\Omega), y_{n}=\sum_{1}^{n} \omega_{i}^{2} e_{i} \rightarrow u_{1}$ strongly in $L^{2}(\Omega)$.

Considering the following regularized equation of inequality (1.1)

$$
\ddot{\xi}^{n}=M^{n}+N^{n}+h,\left.\quad \xi^{n}\right|_{t=0}=\omega^{1 n},\left.\quad \dot{\xi}^{n}\right|_{t=0}=\omega^{2 n}
$$

where $\xi^{n}=\left\{\xi_{i}^{n}\right\}_{1 \times n}, \omega^{1 n}=\left\{\omega_{i}^{1}\right\}_{1 \times n}, \omega^{2 n}=\left\{\omega_{i}^{2}\right\}_{1 \times n}, h=\left\{\left\langle f, e_{i}\right\rangle\right\}_{1 \times n}, M^{n}=\left\{M_{i}^{n}\right\}_{1 \times n}$, $M_{i}^{n}=-\left(A(t)\left(\sum_{1}^{n} \dot{\xi}_{j}^{n} e_{j}\right), e_{i}\right), N^{n}=\left\{N_{i}^{n}\right\}_{1 \times n}, N_{i}^{n}=-\left\langle B\left(\sum_{1}^{n} \xi_{j}^{n} e_{j}\right), e_{i}\right\rangle-\left\langle b_{n}\left(\sum_{1}^{n} \xi_{j}^{n} e_{j}\right), e_{i}\right\rangle$, where "." denotes time derivate.

Equation (3.3) is a vector-valued ordinary differential equation and its local solution $\xi^{n}$ exists on $I_{n}=\left[0, T_{n}\right], 0<T_{n} \leq T$. Set $u_{n}(t)=\sum_{1}^{n} \xi_{j}^{n} e_{j}\left(t \in I_{n}\right)$. Equation (3.3) is equal to

$$
\left\langle\ddot{u}_{n}, e_{i}\right\rangle=-\left(A(t) \dot{u}_{n}, e_{i}\right)-\left\langle B u_{n}, e_{i}\right\rangle-\left\langle b_{n}\left(u_{n}\right), e_{i}\right\rangle+\left\langle f, e_{i}\right\rangle, \quad i=1,2, \ldots, n .
$$

Multiplying (3.4) by $\dot{\xi}_{i}^{n}$, summing over from $i=1$ to $i=n$ and integrating over $[0, t]$ $\left(t \leq I_{n}\right)$, we get

$$
\begin{aligned}
\left|\dot{u}_{n}(t)\right|_{L^{2}(\Omega)}^{2}+\left\langle B u_{n}(t), u_{n}(t)\right\rangle+2 \int_{0}^{t}\left(A \dot{u}_{n}, \dot{u}_{n}\right) d \tau+2 \int_{0}^{t}\left\langle b_{n}\left(u_{n}\right), \dot{u}_{n}\right\rangle d \tau \\
=2 \int_{0}^{t}\left\langle f, \dot{u}_{n}\right\rangle d \tau+\left(y_{n}, y_{n}\right)+\left\langle B x_{n}, x_{n}\right\rangle,
\end{aligned}
$$


but

$$
\begin{aligned}
\int_{0}^{t}\left\langle b_{n}\left(u_{n}\right), \dot{u}_{n}\right\rangle d \tau & =\left.\int_{\Omega} J\left(u_{n}(x, \tau)\right)\right|_{0} ^{t} d x \\
& =\int_{\Omega}\left\{\int_{0}^{u_{n}(x, t)} b_{n}(\lambda) d \lambda-\int_{0}^{u_{n}(x, 0)} b_{n}(\lambda) d \lambda\right\} d x, \\
\left|\int_{0}^{t}\left\langle b_{n}\left(u_{n}\right), \dot{u}_{n}\right\rangle d \tau\right| & \leq c \int_{\Omega}\left\{\left|u_{n}(x, t)\right|+\left|u_{n}(x, 0)\right|+\left|\int_{0}^{u_{n}(x, t)}\right| \lambda|d \lambda|\right. \\
& \left.+\left|\int_{0}^{u_{n}(x, 0)}\right| \lambda|d \lambda|\right\} d x, \\
\left|\int_{0}^{u_{n}(x, t)}\right| \lambda|d \lambda| & =\frac{1}{2}\left|u_{n}(x, t)\right|^{2}, \\
\left|\int_{0}^{u_{n}(x, 0)}\right| \lambda|d \lambda| & =\frac{1}{2}\left|u_{n}(x, 0)\right|^{2}, \\
\left|\int_{0}^{t}\left\langle b_{n}\left(u_{n}\right), \dot{u}_{n}\right\rangle d \tau\right| & \leq \frac{c}{2}(1+|\Omega|)\left\{\left|u_{n}(t)\right|_{L^{2}(\Omega)}^{2}+\left|x_{n}\right|_{L^{2}(\Omega)}^{2}\right\},
\end{aligned}
$$

where $|\Omega|$ denotes the Lebesgue measure of the domain $\Omega$.

From (3.5), it follows that there exists $c_{4}>0$ such that

$$
\left|\dot{u}_{n}(t)\right|_{L^{2}(\Omega)}^{2}+\left|u_{n}(t)\right|_{H_{0}^{1}(\Omega)}^{2} \leq c_{4}+\left\{c_{3}+\frac{c}{2}(1+|\Omega|)\right\}\left|u_{n}(t)\right|_{L^{2}(\Omega)}^{2}+2 \int_{0}^{t}\left\langle f, \dot{u}_{n}\right\rangle d \tau .
$$

We note that

$$
\begin{gathered}
u_{n}(t)=u_{n}(0)+\int_{0}^{t} \dot{u}_{n} d \tau \\
\left|u_{n}(t)\right|_{L^{2}(\Omega)}^{2} \leq\left|u_{n}(0)\right|_{L^{2}(\Omega)}+\int_{0}^{t}\left|\dot{u}_{n}\right|_{L^{2}(\Omega)} d \tau,
\end{gathered}
$$

using Hölder's inequality, we get that there exists $c_{5}, c_{6}>0$ such that

$$
\begin{aligned}
\left|u_{n}(t)\right|_{L^{2}(\Omega)}^{2} & \leq c_{5}+c_{6} \int_{0}^{t}\left|\dot{u}_{n}\right|_{L^{2}(\Omega)}^{2} d \tau \\
\int_{0}^{t}\left\langle f, \dot{u}_{n}\right\rangle d \tau & \leq|f|_{L^{2}\left(0, T ; L^{2}(\Omega)\right)} \cdot\left|\dot{u}_{n}\right|_{L^{2}\left(0, t ; L^{2}(\Omega)\right)} \\
& \leq \frac{1}{2}\left(|f|_{L^{2}\left(0, T ; L^{2}(\Omega)\right)}^{2}+\left|\dot{u}_{n}\right|_{L^{2}\left(0, t ; L^{2}(\Omega)\right)}^{2}\right) .
\end{aligned}
$$

From (3.7), (3.9), and (3.10), we obtain that there exists $c_{7}, c_{8}>0$ such that

$$
\left|\dot{u}_{n}(t)\right|_{L^{2}(\Omega)}^{2}+c_{2}\left|u_{n}(t)\right|_{H_{0}^{1}(\Omega)}^{2} \leq c_{7}+c_{8} \int_{0}^{t}\left|\dot{u}_{n}(\tau)\right|_{L^{2}(\Omega)}^{2} d \tau \quad\left(t \in I_{n}\right),
$$

this implies that

$$
\left|\dot{u}_{n}(t)\right|_{L^{2}(\Omega)}^{2} \leq c_{7}+c_{8} \int_{0}^{t}\left|\dot{u}_{n}(\tau)\right|_{L^{2}(\Omega)}^{2} d \tau \quad\left(t \in I_{n}\right) .
$$

Using Gronwall's inequality it follows that

$$
\left|\dot{u}_{n}(t)\right|_{L^{2}(\Omega)}^{2} \leq c_{7} \exp \left(c_{8} t\right) \quad\left(t \in I_{n}\right) .
$$


Therefore, from (3.9), (3.11), and (3.13), we get that there exists $c_{9}>0$,

$$
\left|\dot{u}_{n}(t)\right|_{L^{2}(\Omega)} \leq c_{9}, \quad\left|u_{n}(t)\right|_{L^{2}(\Omega)} \leq c_{9}, \quad\left|u_{n}(t)\right|_{H_{0}^{1}(\Omega)} \leq c_{9}, \quad\left(t \in I_{n}\right),
$$

where $c_{4}, c_{5}, c_{6}, c_{7}, c_{8}, c_{9}$ are positive constants independent of $n$ and $T_{n}$, from which we can assert that $I_{n}=[0, T](\forall n)$.

For every $\eta \in \operatorname{span}\left\{e_{1}, e_{2}, \ldots, e_{n}\right\}$, from (3.4)

$$
\begin{aligned}
\left|\left\langle\ddot{u}_{n}, \eta\right\rangle\right| \leq & \left|A(t)\left(\dot{u}_{n}\right)\right|_{L^{2}(\Omega)} \cdot|\eta|_{L^{2}(\Omega)}+|f(t)|_{L^{2}(\Omega)} \cdot|\eta|_{L^{2}(\Omega)} \\
& +\left|b_{n}\left(u_{n}\right)\right|_{L^{2}(\Omega)} \cdot|\eta|_{L^{2}(\Omega)}+|B| \cdot\left|u_{n}\right|_{H_{0}^{1}(\Omega)} \cdot|\eta|_{H_{0}^{1}(\Omega)},
\end{aligned}
$$

where $|B|$ is the norm of linear continuous operator $B$

$$
\begin{aligned}
& \left|\ddot{u}_{n}(t)\right|_{H^{-1}(\Omega)} \\
& \quad=\sup _{|\eta|_{V}=1}\left|\left\langle\ddot{u}_{n}(t), \eta\right\rangle\right|=\sup _{\substack{\left.\eta \in \operatorname{span}_{\left.|\eta|_{1}, \ldots, e_{n}\right\}}|| \eta\right|_{V}=1}}\left|\left\langle\ddot{u}_{n}(t), \eta\right\rangle\right| \\
& \quad \leq c_{10}\left(\left|A(t)\left(\dot{u}_{n}\right)\right|_{L^{2}(\Omega)}+|f(t)|_{L^{2}(\Omega)}+\left|b_{n}\left(u_{n}\right)\right|_{L^{2}(\Omega)}\right)+|B| \cdot\left|u_{n}(t)\right|_{H_{0}^{1}(\Omega)},
\end{aligned}
$$

where $c_{10}$ is the imbedding constant which $H_{0}^{1}(\Omega)$ imbeds in $L^{2}(\Omega)$

$$
\begin{aligned}
\left|b_{n}\left(u_{n}\right)(t)\right|_{L^{2}(\Omega)}^{2} & =\int_{\Omega}\left|b_{n}\left(u_{n}\right)(t)\right|^{2} d x \leq \int_{\Omega} c^{2}\left(1+\left|u_{n}(x, t)\right|\right)^{2} d x \\
& \leq 2 c^{2} \int_{\Omega}\left(1+\left|u_{n}(x, t)\right|^{2}\right) d x=2 c^{2}\left(|\Omega|+\left|u_{n}(t)\right|_{L^{2}(\Omega)}^{2}\right),
\end{aligned}
$$

this shows that $\left\{b_{n}\left(u_{n}\right)\right\}$ is also a bounded subset of $L^{\infty}\left(0, T ; L^{2}(\Omega)\right)$. Since $A$ is weakly continuous, it must be a bounded operator from $L^{2}\left(0, T ; L^{2}(\Omega)\right)$ to $L^{2}\left(0, T ; L^{2}(\Omega)\right)$. But $\left\{\dot{u}_{n}\right\}$ is a bounded subset of $L^{2}\left(0, T ; L^{2}(\Omega)\right),\left\{A(t)\left(\dot{u}_{n}\right)\right\}$ must be a bounded subset of $L^{2}\left(0, T ; L^{2}(\Omega)\right)$. Inequality (3.16) implies that $\left\{\ddot{u}_{n}\right\}$ is a bounded subset of $L^{2}\left(0, T ; H^{-1}(\Omega)\right)$.

Therefore, there exist a subsequence of $\left\{u_{n}\right\}$, still denoted by itself, and a function $u$ such that $u \in L^{\infty}\left(0, T ; H_{0}^{1}(\Omega)\right), \dot{u} \in L^{\infty}\left(0, T ; L^{2}(\Omega)\right), \ddot{u} \in L^{2}\left(0, T ; H^{-1}(\Omega)\right)$ satisfying

$$
\begin{aligned}
& u_{n} \rightarrow u \quad \text { weakly-star in } L^{\infty}\left(0, T ; H_{0}^{1}(\Omega)\right), \\
& \dot{u}_{n} \longrightarrow \dot{u} \text { weakly-star in } L^{\infty}\left(0, T ; L^{2}(\Omega)\right), \\
& \ddot{u}_{n} \longrightarrow \ddot{u} \text { weakly in } L^{2}\left(0, T ; L^{-1}(\Omega)\right), \\
& b_{n}\left(u_{n}\right) \rightarrow g \quad \text { weakly-star in } L^{\infty}\left(0, T ; L^{2}(\Omega)\right) .
\end{aligned}
$$

Since, the space $W(V)$ defined by $W(V)=\left\{u \in L^{2}(0, T ; V), \dot{u} \in L^{2}\left(0, T ; V^{\prime}\right)\right\}$ forms a real Hilbert space with the norm $|u|_{W}=|u|_{L^{2}(0, T ; V)}+|\dot{u}|_{L^{2}\left(0, T ; V^{\prime}\right)}$ and is continuously imbedded in $C\left([0, T] ; L^{2}(\Omega)\right)$, it is obvious that $u \in C\left(0, T ; L^{2}(\Omega)\right), \dot{u} \in C\left(0, T ; H^{-1}(\Omega)\right)$. Hence, $u(0), \dot{u}(0)$ make sense.

For $\lambda \in L^{2}(0, T)$, from (3.4) we have

$$
\begin{aligned}
\int_{0}^{T}\left\langle\ddot{u}_{n}, \lambda e_{i}\right\rangle d t= & -\int_{0}^{T}\left(A(t)\left(\dot{u}_{n}\right), \lambda e_{i}\right) d t-\int_{0}^{T}\left\langle B\left(u_{n}\right), \lambda e_{i}\right\rangle d t \\
& -\int_{0}^{T}\left\langle b_{n}\left(u_{n}\right), \lambda e_{i}\right\rangle d t+\int_{0}^{T}\left\langle f(t), \lambda e_{i}\right\rangle d t, \quad i=1,2, \ldots, n .
\end{aligned}
$$


For every given positive integer $i$, let $n \rightarrow \infty$ in (3.19), it follows that

$$
\begin{aligned}
\int_{0}^{T}\left\langle\ddot{u}, \lambda e_{i}\right\rangle d t= & -\int_{0}^{T}\left(A(t)(\dot{u}), \lambda e_{i}\right) d t-\int_{0}^{T}\left\langle B(u), \lambda e_{i}\right\rangle d t \\
& -\int_{0}^{T}\left\langle g, \lambda e_{i}\right\rangle d t+\int_{0}^{T}\left\langle f(t), \lambda e_{i}\right\rangle d t, \quad i=1,2, \ldots, n .
\end{aligned}
$$

Therefore, we have from (3.20)

$$
\ddot{u}(t)+A(t)(\dot{u})+B(u)+g(t)=f(t), \quad \text { in } L^{2}\left(0, T ; H^{-1}(\Omega)\right) .
$$

Next, we demonstrate that

$$
g(x, t) \in \varphi(u(x, t)) \text { a.e. }(x, t) \in Q_{T}=\Omega \times[0, T] .
$$

Since, $u_{n}(x, t) \rightarrow u(x, t)$ a.e. $(x, t) \in Q_{T}$, by Eropoß's theorem [9], for every $\delta>0$, there exists a subset $Q_{\delta} \subseteq Q_{T}=\Omega \times[0, T],\left|Q_{\delta}\right| \leq \delta$,

$$
u_{n}(x, t) \longrightarrow u(x, t) \quad \text { uniformly in } Q_{T} \backslash Q_{\delta}
$$

that is, for every $\varepsilon>0$, there exists a positive integer $\bar{N}$, when $n \geq \bar{N}$,

$$
\left|u_{n}(x, t)-u(x, t)\right| \leq \varepsilon \quad \forall(x, t) \in Q_{T} \backslash Q_{\delta} .
$$

It is obvious that, when $1 / n \leq \varepsilon$ and $n \geq \bar{N}$, for almost everywhere $(x, t) \in Q_{T} \backslash Q_{\delta}$

$$
\underline{b}_{n}\left(u_{n}(x, t)\right)=b_{n}\left(u_{n}(x, t)\right)=\bar{b}_{n}\left(u_{n}(x, t)\right) \leq \bar{b}_{\varepsilon}\left(u_{n}(x, t)\right) \leq \bar{b}_{2 \varepsilon}(u(x, t)) .
$$

For every $\mu \in L^{1}\left(0, T ; L^{2}(\Omega)\right), \mu \geq 0$

$$
\begin{aligned}
\int_{Q_{T} \backslash Q_{\delta}} g(x, t) \mu(x, t) d x d t & =\lim _{n \rightarrow \infty} \int_{Q_{T} \backslash Q_{\delta}} b_{n}\left(u_{n}(x, t)\right) \mu(x, t) d x d t \\
& \leq \int_{Q_{T} \backslash Q_{\delta}} \bar{b}_{2 \varepsilon}(u(x, t)) \mu(x, t) d x d t, \\
\int_{Q_{T} \backslash Q_{\delta}} g(x, t) \mu(x, t) d x d t & \leq \limsup _{\varepsilon \rightarrow 0^{+}} \int_{Q_{T} \backslash Q_{\delta}} \bar{b}_{2 \varepsilon}(u(x, t)) \mu(x, t) d x d t \\
& \leq \int_{Q_{T} \backslash Q_{\delta}} \bar{b}(u(x, t)) \mu(x, t) d x d t .
\end{aligned}
$$

Analogously, we can obtain

$$
\int_{Q_{T} \backslash Q_{\delta}} g(x, t) \mu(x, t) d x d t \geq \int_{Q_{T} \backslash Q_{\delta}} \underline{b}(u(x, t)) \mu(x, t) d x d t .
$$

Hence, (3.26) and (3.27) imply that

$$
g(x, t) \in \varphi(u(x, t)) \text { a.e. }(x, t) \in Q_{T} \backslash Q_{\delta} .
$$

Finally, let $\delta \rightarrow 0^{+}$, we get

$$
g(x, t) \in \varphi(u(x, t)) \text { a.e. }(x, t) \in Q_{T}=\Omega \times[0, T] .
$$


Let $\lambda \in C^{1}[0, T], \lambda(T)=0$, integrating by parts the left-hand side of equations (3.19) and (3.20) gives

$$
\begin{aligned}
-\left\langle\dot{u}_{n}(0), \lambda(0) e_{i}\right\rangle-\int_{0}^{T}\left\langle\dot{u}_{n}, \dot{\lambda} e_{i}\right\rangle d t= & -\int_{0}^{T}\left(A(t)\left(\dot{u}_{n}\right), \lambda e_{i}\right) d t-\int_{0}^{t}\left\langle B\left(u_{n}\right), \lambda e_{i}\right\rangle d t \\
& -\int_{0}^{T}\left\langle b_{n}\left(u_{n}\right), \lambda e_{i}\right\rangle d t-\int_{0}^{T}\left\langle f(t), \lambda e_{i}\right\rangle d t, \\
-\left\langle\dot{u}(0), \lambda(0) e_{i}\right\rangle-\int_{0}^{T}\left\langle\dot{u}, \dot{\lambda} e_{i}\right\rangle d t= & -\int_{0}^{T}\left(A(t)(\dot{u}), \lambda e_{i}\right) d t-\int_{0}^{t}\left\langle B(u), \lambda e_{i}\right\rangle d t \\
& -\int_{0}^{T}\left\langle g, \lambda e_{i}\right\rangle d t-\int_{0}^{T}\left\langle f(t), \lambda e_{i}\right\rangle d t,
\end{aligned}
$$

making comparison between (3.30) and (3.31) we get that

$$
\lim _{n \rightarrow \infty}\left\langle\dot{u}_{n}(0)-\dot{u}(0), e_{i}\right\rangle=0, \quad i=1,2, \ldots, n
$$

therefore, this implies that

$$
\dot{u}_{n}(0) \longrightarrow \dot{u}(0) \quad \text { weakly in } H^{-1}(\Omega)
$$

uniqueness of limit implies that $\dot{u}(0)=u_{1}$ (in $H^{-1}(\Omega)$ ).

Let $\lambda \in C^{2}[0, T], \lambda(T)=0, \dot{\lambda}(T)=0$. Analogously, integrating by parts the left-hand side of equations (3.30) and (3.31), and making comparison with the obtained results again gives: $u(0)=u_{0}\left(\right.$ in $L^{2}(\Omega)$ ).

THEOREM 3.2. Let $f \in L^{2}\left(0, T ; L^{2}(\Omega)\right), u_{0} \in H_{0}^{1}(\Omega) \cap L^{\infty}(\Omega), u_{1} \in L^{2}(\Omega)$. Assume that $b$ satisfies

$\left(1^{\prime}\right) b(\xi) \xi \geq 0$ for almost everywhere $\xi \in \mathbb{R}$, and $\exists \bar{c}>0$,

$$
|b(\xi)| \leq \bar{c}\left(1+|\xi|^{p}\right) \text {, a.e. } \xi \in \mathbb{R} \text {, if } n>2,0<p \leq \frac{2 n}{n-2} \text {; if } n \leq 2,0 \leq p<\infty \text {. }
$$

Then, under assumptions (2), (3), and (4), there exists a function $v$ defined in $\Omega \times[0, T]$ satisfying

$$
\begin{gathered}
v \in L^{\infty}\left(0, T ; H_{0}^{1}(\Omega)\right), \quad \dot{v} \in L^{\infty}\left(0, T ; L^{2}(\Omega)\right), \\
\ddot{v}+A(t)(\dot{v})+B(v)+\bar{g}(t)=f(t) \quad \text { in } L^{1}\left(0, T ; H^{-1}(\Omega)+L^{1}(\Omega)\right), \\
\bar{g}(x, t) \in \varphi(v(x, t)) \quad \text { a.e. }(x, t) \in Q_{T}=\Omega \times[0, T], \\
v(0)=u_{0}, \quad \dot{v}(0)=u_{1} .
\end{gathered}
$$

Proof. It is also easy to see that $b_{\varepsilon}$ satisfies assumption $(1)^{\prime}$ with possible different constant $\bar{c}$. Analogously to Theorem 3.1 , we still may get (3.5), where $\left\{e_{n}\right\}_{n=1}^{\infty}$ is a basis of $H_{0}^{1}(\Omega) \cap L^{\infty}(\Omega)$ satisfying $\left(e_{i}, e_{j}\right)=\delta_{i j}$. Set

$$
J_{n}(\xi)=\int_{0}^{\xi} b_{n}(t) d t
$$

then $J_{n}(\xi) \geq 0, \forall \xi \in \mathbb{R}$, and

$$
\begin{aligned}
& \int_{0}^{t}\left\langle b_{n}\left(u_{n}\right), \dot{u}_{n}\right\rangle d \tau=\int_{\Omega} J_{n}\left(u_{n}(x, t)\right) d x-\int_{\Omega} J_{n}\left(u_{n}(x, 0)\right) d x \geq-\int_{\Omega} J_{n}\left(u_{n}(x, t)\right) d x \\
&\left|b_{n}(\xi)\right| \leq\left.\left.\left|\int_{|z| \leq 1} \beta(z)\right| b\left(\xi-\frac{z}{n}\right)\right|^{p} d z\left|\leq d_{1}+d_{2}\right| \xi\right|^{p},
\end{aligned}
$$


where $d_{1}$ and $d_{2}$ are positive constants independent of $n$.

$$
\begin{aligned}
\left|J_{n}\left(x_{n}\right)\right| & =\left|\int_{0}^{x_{n}} b_{n}(t) d t\right| \leq\left(\operatorname{sgn} x_{n}\right) \cdot \int_{0}^{x_{n}}\left|b_{n}(t)\right| d t \\
& \leq\left(\operatorname{sgn} x_{n}\right) \cdot \int_{0}^{x_{n}}\left(d_{1}+d_{2}|t|^{p}\right) d t=d_{1}\left|x_{n}\right|+\frac{d_{2}\left|x_{n}\right|^{p+1}}{(p+1)}, \\
\left|\int_{\Omega} J_{n}\left(x_{n}\right) d t\right| & \leq \int_{\Omega}\left|J_{n}\left(x_{n}\right)\right| d x \leq d_{1}\left|x_{n}\right|_{L^{1}(\Omega)}+\frac{d_{2}\left|x_{n}\right|_{L^{p+1}(\Omega)}^{p+1}}{(p+1)} .
\end{aligned}
$$

Since $L^{p+1}(\Omega) \subset L^{1}(\Omega)$ and $u_{n}(0)=x_{n} \rightarrow u_{0}$ strongly in $L^{p+1}(\Omega)$, and $\left|x_{n}\right|_{L^{1}(\Omega)}$ are bounded, and so is $\int_{\Omega} J_{n}\left(x_{n}(x)\right) d x$. From (3.5) we have

$$
\left|\dot{u}_{n}(t)\right|_{L^{2}(\Omega)}^{2}+c_{2}\left|u_{n}(t)\right|_{H_{0}^{1}(\Omega)}^{2} \leq c_{4}+c_{3}\left|u_{n}(t)\right|_{L^{2}(\Omega)}^{2}+2 \int_{0}^{t}\left\langle f, \dot{u}_{n}\right\rangle d \tau .
$$

It is easy to see that (3.9), (3.10), (3.11), (3.13), and (3.14) are still true and the solution of (3.3) can be extended to interval $[0, T]$. By Sobolev imbedding theorem, we have, for a.e. $t \in[0, T]$, if $n>2$, then $H_{0}^{1}(\Omega) \subset L^{p^{*}}(\Omega) \subset L^{p}(\Omega), p^{*}=2 n /(n-2)$, and $\left|u_{n}(t)\right|_{L^{p}(\Omega)} \leq c_{10}\left|u_{n}(t)\right|_{H_{0}^{1}(\Omega)} \leq c_{10} c_{9}$; if $n=2$, then $H_{0}^{1}(\Omega) \subset L^{q}(\Omega)$, when $1 \leq q<\infty$, so $\left|u_{n}(t)\right|_{L^{p}(\Omega)} \leq c_{10}\left|u_{n}(t)\right|_{H_{0}^{1}(\Omega)} \leq c_{10} c_{9}$; if $n=1$, then $H_{0}^{1}(\Omega) \subset C(\bar{\Omega})$ and ditto, $\left|u_{n}(t)\right|_{C(\bar{\Omega})}=\max _{x \in \bar{\Omega}}\left|u_{n}(x, t)\right| \leq c_{10} c_{9}$, where $\bar{\Omega}$ denotes the closure of $\Omega$ and $c_{10}$ is the imbedding constant which $H_{0}^{1}(\Omega)$ imbeds in $L^{p}(\Omega)$ or $C(\bar{\Omega})$. Note that, we always have that $b_{n}\left(u_{n}\right) \in L^{\infty}\left(0, T ; L^{p_{0}}(\Omega)\right)$, where $p_{0}=(n+1) /(n-2)$ and $\left\{b_{n}\left(u_{n}\right)\right\}$ is a bounded subset of $L^{\infty}\left(0, T ; L^{p_{0}}(\Omega)\right)$. Therefore, there exist a subsequence of $\left\{u_{n}\right\}$, still denoted by itself, and a function $v$ such that $v \in L^{\infty}\left(0, T ; H_{0}^{1}(\Omega)\right), \dot{v} \in L^{\infty}\left(0, T ; L^{2}(\Omega)\right)$ satisfying

$$
\begin{aligned}
& u_{n} \longrightarrow v \quad \text { weakly-star in } L^{\infty}\left(0, T ; H_{0}^{1}(\Omega)\right), \\
& \dot{u}_{n} \longrightarrow \dot{v} \quad \text { weakly-star in } L^{\infty}\left(0, T ; L^{2}(\Omega)\right), \\
& b_{n}\left(u_{n}\right) \rightarrow g \quad \text { weakly-star in } L^{\infty}\left(0, T ; L^{p_{0}}(\Omega)\right) .
\end{aligned}
$$

Since, the dual of the space $H_{0}^{1}(\Omega) \cap L^{\infty}(\Omega)$ is the space $L^{1}\left(0, T ; H^{-1}(\Omega)+L^{1}(\Omega)\right)$, it is easy to obtain from (3.4) that

$$
\ddot{v}(t)+A(t) \dot{v}+B(v)+\bar{g}(t)=f(t) \quad \text { in } L^{1}\left(0, T ; H^{-1}(\Omega)+L^{1}(\Omega)\right) .
$$

Analogous to Theorem 3.1, we can complete the proof of this theorem.

REMARK 3.3. If $A(t)=A$ and $A$ is linear, then the uniqueness of such solution will be obtained in the same way as in [3].

\section{Decay of the solution}

THEOREM 4.1. Let $T=+\infty, f \equiv 0$. Suppose that for every $t \geq 0$, the operator $A(t)$ satisfies

$$
(A(t) w, w) \geq \delta_{0}|w|_{L^{2}(\Omega)}^{2}, \quad \forall w \in L^{2}(\Omega) .
$$

Moreover, if $\langle B w, w\rangle \geq 0, \forall w \in H_{0}^{1}(\Omega)$ or $c_{3} c_{10}^{2} \leq c_{2}$, here $c_{10}$ is an imbedding constant which $H_{0}^{1}(\Omega)$ imbeds in $L^{2}(\Omega)$. Then, under conditions of Theorem 3.2, the solution in 
Theorem 3.2 obtained from the regularized equation (3.3) satisfies

$$
|\dot{u}(t)|_{L^{2}(\Omega)}^{2} \leq \mu_{1} \exp \left(-\mu_{2} t\right), \text { a.e. } t \geq 0,
$$

where $\delta_{0}, \mu_{1}, \mu_{2}$ are positive constants.

Proof. Let $u_{n}$ be a solution of (3.3), that is, satisfies (3.4) and (3.5). Since $J_{n}\left(u_{n}(x, t)\right) \geq 0$, by (3.5) we have

$$
|\dot{u}(t)|_{L^{2}(\Omega)}^{2}+\left\langle B u_{n}(t), u_{n}(t)\right\rangle \leq c_{11}-2 \delta_{0} \int_{0}^{t}\left|\dot{u}_{n}(\tau)\right|_{L^{2}(\Omega)}^{2} d \tau, \quad t \in[0,+\infty),
$$

where $c_{11}$ is a positive constant independent of $n$. If $\langle B w, w\rangle \geq 0$, for every $w \in H_{0}^{1}(\Omega)$, $\left\langle B u_{n}(t), u_{n}(t)\right\rangle \geq 0$. Analogously to [7, Theorem 4], we obtain:

$$
\left|\dot{u}_{n}(t)\right|_{L^{2}(\Omega)}^{2} \leq c_{11} \exp \left(-2 \delta_{0} t\right), \quad \text { a.e. } t \geq 0 .
$$

If $c_{3} c_{10}^{2} \leq c_{2}$, we get from (4.3) that

$$
\begin{aligned}
\left|\dot{u}_{n}(t)\right|_{L^{2}(\Omega)}^{2}+c_{2}\left|u_{n}(t)\right|_{H_{0}^{1}(\Omega)}^{2} & \leq c_{11}+c_{3}\left|u_{n}(t)\right|_{L^{2}(\Omega)}^{2}-2 \delta_{0} \int_{0}^{t}\left|\dot{u}_{n}(\tau)\right|_{L^{2}(\Omega)}^{2} d \tau \\
& \leq c_{11}+c_{3} c_{10}^{2}\left|u_{n}(t)\right|_{H_{0}^{1}(\Omega)}^{2}-2 \delta_{0} \int_{0}^{t}\left|\dot{u}_{n}(\tau)\right|_{L^{2}(\Omega)}^{2} d \tau
\end{aligned}
$$

from which it is permitted to get inequality (4.4).

Since $\left|\dot{u}_{n}(t)\right|_{L^{2}(\Omega)} \leq c_{9}, \dot{u} \rightarrow \dot{u}$ weakly-star in $L^{\infty}\left(0, \infty ; L^{2}(\Omega)\right)$, it is easy to obtain that $\dot{u}(t) \rightarrow \dot{u}(t)$ weak in $L^{2}(\Omega)$ for a.e. $t \geq 0$. But $L^{2}(\Omega)$ is a real Hilbert space, therefore, $|\dot{u}(t)|_{L^{2}(\Omega)} \leq \underline{\lim }_{n \rightarrow \infty}\left|\dot{u}_{n}(t)\right|_{L^{2}(\Omega)}$, a.e. $t \geq 0$. Finally, we get $|\dot{u}(t)|_{L^{2}(\Omega)}^{2} \leq$ $c_{11} \exp \left(-2 \delta_{0} t\right)$, (a.e. $\left.t \geq 0\right)$.

\section{REFERENCES}

[1] S. Carl and S. Heikkilä, An existence result for elliptic differential inclusions with discontinuous nonlinearity, Nonlinear Anal. 18 (1992), no. 5, 471-479. MR 92m:35268. Zbl 755.35039 .

[2] K. C. Chang, Variational methods for nondifferentiable functionals and their applications to partial differential equations, J. Math. Anal. Appl. 80 (1981), no. 1, 102-129. MR 82h:35025. Zbl 487.49027.

[3] M. R. Clark, Existence of solutions for a nonlinear hyperbolic-parabolic equation in a noncylinder domain, Int. J. Math. Math. Sci. 19 (1996), no. 1, 151-160. MR 96h:35073. Zbl 842.35062.

[4] D. G. Costa and J. V. A. Gonçalves, Critical point theory for nondifferentiable functionals and applications, J. Math. Anal. Appl. 153 (1990), no. 2, 470-485. MR 91j:58034. Zbl 717.49007.

[5] O. A. de Lima, Existence and uniqueness of solutions for an abstract nonlinear hyperbolicparabolic equation, Appl. Anal. 24 (1987), no. 1-2, 101-116. MR 88i:34124. Zbl 589.35063.

[6] J. Ferreira, On weak solutions of semilinear hyperbolic-parabolic equations, Int. J. Math. Math. Sci. 19 (1996), no. 4, 751-758. MR 97b:35123. Zbl 861.35062.

[7] X. Guo, On existence and uniqueness of solution of hyperbolic differential inclusion with discontinuous nonlinearity, J. Math. Anal. Appl. 241 (2000), no. 2, 198-213. CMP 1739 202. Zbl 991.25141. 
[8] N. A. Lar'kin, Boundary value problems in the large for a class of hyperbolic equations, Sibirsk. Mat. Ž. 18 (1977), no. 6, 1414-1419, p. 1438 (Russian). MR 58\#17542.

[9] J.-L. Lions, Quelques méthodes de résolution des problèmes aux limites non linéaires, Dunod, Gauthier-Villars, Paris, 1969 (French). MR 41\#4326. Zbl 189.40603.

[10] A. B. Maciel, On hyperbolic-parabolic equation with a continuous nonlinearity, Nonlinear Anal. 20 (1993), no. 6, 745-754. MR 94f:35090. Zbl 802.35060.

[11] Z. Naniewicz and P. D. Panagiotopoulos, Mathematical Theory of Hemivariational Inequalities and Applications, Monographs and Textbooks in Pure and Applied Mathematics, vol. 188, Marcel Dekker, Inc., New York, 1995. MR 96d:47067. Zbl 950.05618.

[12] P. D. Panagiotopoulos, Inequality Problems in Mechanics and Applications, Convex and Nonconvex Energy Functions, Birkhäuser Boston, Inc., Boston, Mass., 1985. MR 88h:49003. Zbl 579.73014.

[13]_ Coercive and semicoercive hemivariational inequalities, Nonlinear Anal. 16 (1991), no. 3, 209-231. MR 92m:47138. Zbl 733.49012.

[14] _ Hemivariational Inequalities, Applications in Mechanics and Engineering, Springer-Verlag, Berlin, 1993. MR 97c:73001. Zbl 826.73002.

[15] J. Rauch, Discontinuous semilinear differential equations and multiple valued maps, Proc. Amer. Math. Soc. 64 (1977), no. 2, 277-282. MR 56\#835. Zbl 413.35031.

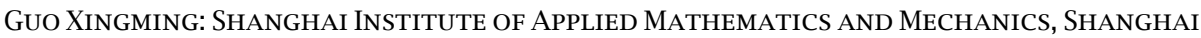
UNIVERSITY, SHANGHAI 200072, CHINA

E-mail address: xmguo@yc.shu.edu.cn 


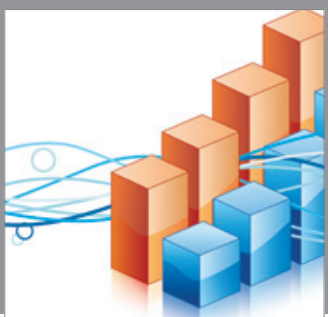

Advances in

Operations Research

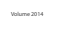

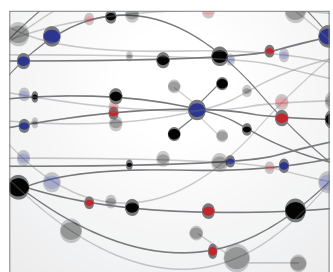

\section{The Scientific} World Journal
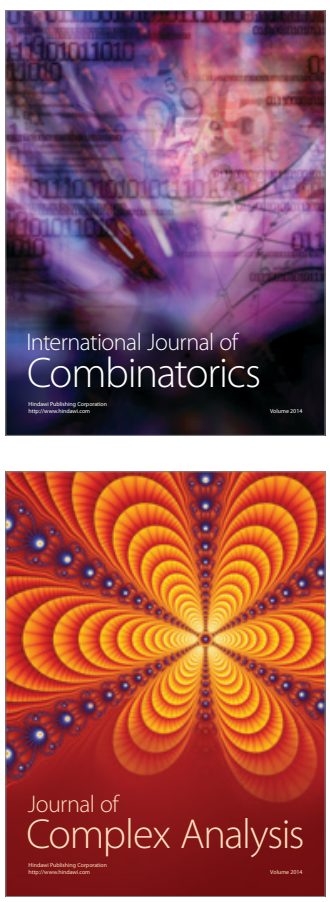

International Journal of

Mathematics and

Mathematical

Sciences
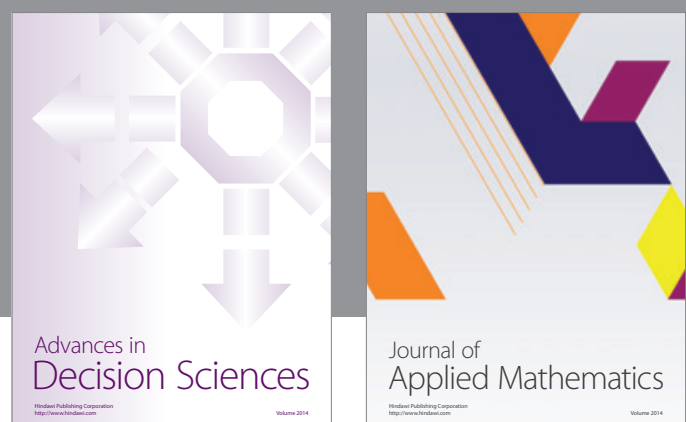

Journal of

Applied Mathematics
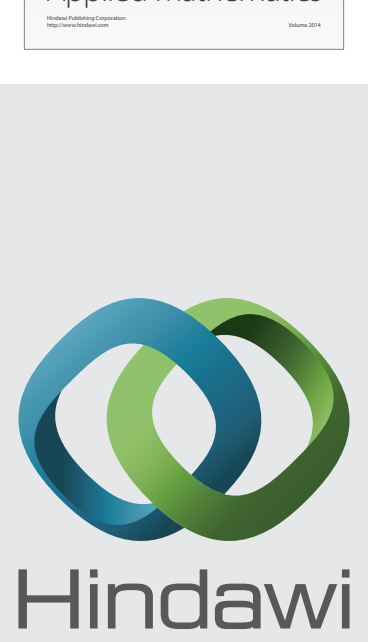

Submit your manuscripts at http://www.hindawi.com
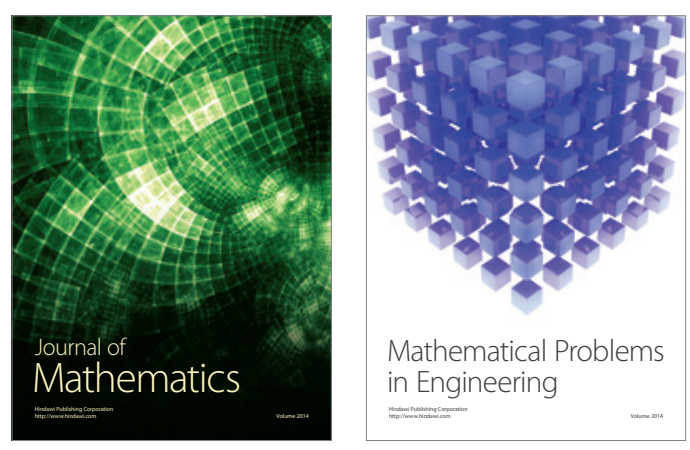

Mathematical Problems in Engineering
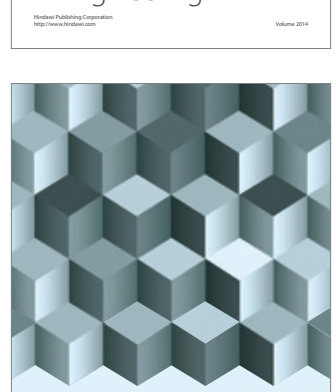

Journal of

Function Spaces
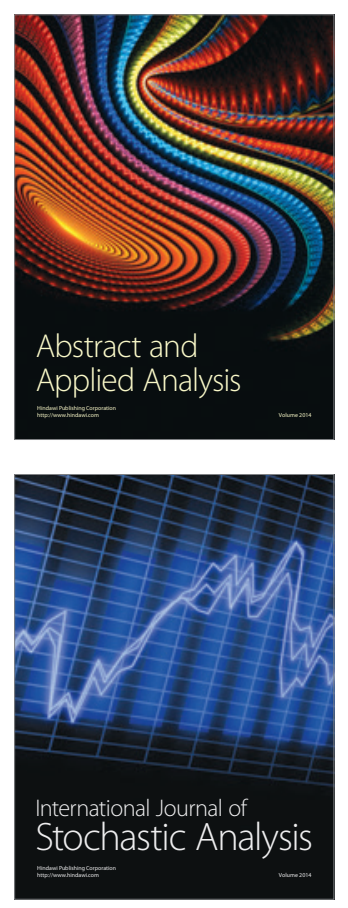

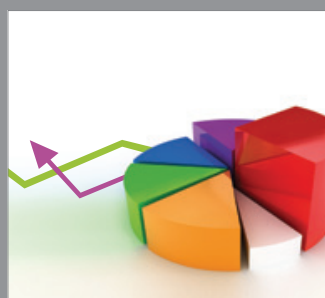

ournal of

Probability and Statistics

Promensencen
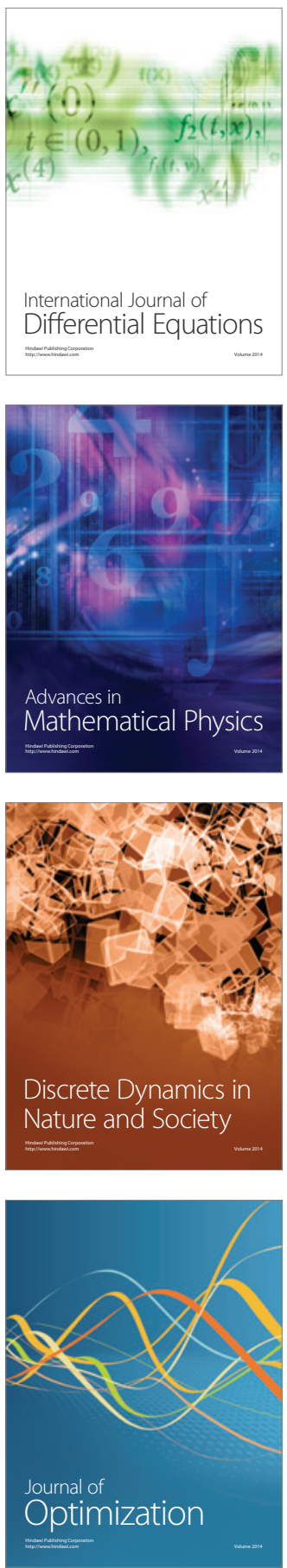